\title{
Muscle Recovery Is Highlighted by IR Laser Therapy
}

\author{
Mohammadreza Razzaghi ${ }^{1}$, Mohammad Rostami-Nejad ${ }^{2}$, Mostafa Rezaei-Tavirani ${ }^{3}$, Mona Zamanian Azodi ${ }^{*}$, \\ Farshad Okhovatian ${ }^{5}$, Vahid Mansouri' ${ }^{3}$, Nayebali Ahmadi ${ }^{3}$
}

\author{
${ }^{1}$ Laser Application in Medical Sciences Research Center, Shahid Beheshti University of Medical Sciences, Tehran, Iran \\ ${ }^{2}$ Gastroenterology and Liver Diseases Research Center, Research Institute for Gastroenterology and Liver Diseases, Shahid \\ Beheshti University of Medical Sciences, Tehran, Iran \\ ${ }^{3}$ Proteomics Research Center, Faculty of Paramedical Sciences, Shahid Beheshti University of Medical Sciences, Tehran, \\ Iran \\ ${ }^{4}$ Proteomics Research Center, Shahid Beheshti University of Medical Sciences, Tehran, Iran \\ ${ }^{5}$ Physiotherapy Research Center, Shahid Beheshti University of Medical Sciences, Tehran, Iran
}

\section{*Correspondence to Mona Zamanian Azodi, Email: mona.azodi@gmail.com \\ Published online December 1,2019}

\begin{abstract}
Introduction: In sports medicine, laser application has been well-established for the recovery of muscles. The mechanisms by which benefits of this kind of therapy can be studied is molecular research approach. Protein-protein interaction network analysis as one of the important complementary studies of proteomics can accelerate this goal by the identification of novel contributing markers.

Methods: By the use of Cytoscape V3.7.1 and its applications, a network of differential expressed proteins (DEPs) from IR laser treatment samples were constructed and analyzed. Six hub-bottlenecks were determined, 4 of which were from differentially expressed proteins.

Results: ClueGO discovered 4 biological processes related to these hub-bottlenecks that their function could alter due to IR laser therapy.

Conclusion: In fact, by the expression changes of hub-bottlenecks including the up-regulation of HSP90s, one of the prominent biological processes in muscle recovery could be activated. This process is called nitric oxide synthase (NOS) activation that could be proposed as one of the underlying mechanisms of IR laser treatments in muscle recovery.

Keywords: Infrared (IR) laser; Myoblast; Proteomics; Protein-protein interaction network analysis
\end{abstract}

\section{Introduction}

Laser therapy has been vastly employed in clinical fields for many years and the subjects including skincare, ${ }^{1}$ surgical methods, ${ }^{2}$ oncology, ${ }^{3}$ and sports medicine. ${ }^{4}$ There are different lines of laser procedures, one of which is infrared laser treatment. The IR laser method has been used for pain-relieving, ${ }^{5}$ dental health, ${ }^{6}$ and sports medicine. ${ }^{4}$

Heavy physical activities could cause some abnormalities in muscles. ${ }^{4}$ As already mentioned, the laser could be beneficial in muscle improvement in a way that the radiation stimulates the cure of wounds and tissues of the traumatized muscles ${ }^{4}$ by the reduction of inflammation. ${ }^{7}$ Molecular investigations including genomics, proteomics, and metabolomics could be helpful to better understand the mechanisms by which the laser could be beneficial in different medical areas. ${ }^{8-10}$ The effect of laser therapy and non-ionized radiation on gene expression has also been reported for Interleukin 6, vascular endothelial growth factor, MyoD and myogenin expressions in a tissue repairing process. ${ }^{7,11}$ Other molecular levels such as proteome evaluations could provide key information regarding the molecular mechanisms related to the biostimulating effect of laser exposure on protein molecules. ${ }^{4}$ Additional information in this relationship could be achieved via the bioinformatic study of molecular data such as proteomics. ${ }^{12}$ Moreover, bioinformatics is a useful method to study mechanism of laser therapy via analyzing the most central biomarkers in an interaction network. ${ }^{8}$ Here, the proteomics data of IR laser treatment on myoblast (C2C12 cell line) of Murine ${ }^{4}$ were employed for our bioinformatic assessments. The protein-protein interaction network analysis of differential expressed proteins (DEPs) obtained from the proteomics investigation was screened for introducing the most essentially contributing biomarkers of the IR Laser mechanism of effects in muscle repair. 


\section{Methods}

In this study, a list of DEPs including 40 spots from "Effect of IR laser on myoblasts: a proteomic study"4 was selected. In the original study, a proteomic evaluation of dual laser InfraRed treatment of the $\mathrm{C} 2 \mathrm{C} 12$ cell line and the skeletal muscle cell, from Murine was handled. Murine satellite cells were the source of the $\mathrm{C} 2 \mathrm{C} 12$ cell line. A multiwave locked system laser (ASA Srl, Vicenza, Italy) was applied; the applied IR laser wavelength was 808 and $905 \mathrm{~nm}$. The main radiation source with average power up to $1.1 \mathrm{~W}$ including two synchronized sources (laser diodes) was applied. The first source is a pulsed laser diode that emits $905 \mathrm{~nm}$ wavelength and a peak optical power of $25 \mathrm{~W}$. The second one was a laser diode $(808 \mathrm{~nm})$ which operated in a continuous mode (power $1.1 \mathrm{~W}$ ) or a pulsed mode, with the mean optical power output of $550 \mathrm{~mW}$.

The plate containing the $\mathrm{C} 2 \mathrm{C} 12$ skeletal muscle cell line was exposed to laser beams with the following scan parameters: $5.6 \mathrm{cms}-1$, each scan time of 20 seconds, and a total scan time of 8 minutes. The treatment was done under sterile conditions once a day for 3 consecutive days.

The regeneration and repair of muscle cells are assigned with these types of cells. The micrographs of the cell line before and after treatment are presented in the original research. ${ }^{4}$ As mentioned earlier, a list of 40 DEPs obtained from IR laser treatments was applied to our bioinformatic approach, the protein-protein interaction network analysis. For this aim, Cytoscape 3.7.1 constructed and analyzed the interaction network via the integrated plugins. ${ }^{13}$ Proteins and their interaction queries were dealt with through the use of the STRING $\mathrm{db}$ application with the confidence cut-off score of 0.4 as the default selection. Four sources are provided through a STRING platform, including STRING PubMed, STRING Protein, STRING Diseases, and STITCH. ${ }^{14}$ The protein queried option was used for the protein-protein interaction network construction. The Uniprot action numbers of 40 proteins were searched against String db. The network was then analyzed for centrality properties including degree $(\mathrm{K})$ and betweenness centrality (BC) as the most important parameters via the Network Analyzer. ${ }^{15}$ Proteins with the highest values of degree were called hubs; similarly, nodes with the highest amount of $\mathrm{BC}$ were named as bottlenecks. Those proteins with the highest scores of $\mathrm{K}$ and $\mathrm{BC}$ were called hub-bottlenecks. Hub-bottlenecks were then studied in terms of enrichment properties. In general, genes and proteins can be assessed for their ontology characteristics, including the biological process (BP), the cellular component (CC), and the molecular function (MF). ClueGO and CluePedia identified the BP related to the Hub-bottlenecks with the following statistical criteria: the grouping cut-off score $=0.5$ and gene ontology $(\mathrm{GO})$ terms; $\mathrm{min}$. number of genes $=1$ and $\mathrm{min}$. gene percentage in term $=3$. The Bonferroni step down was the correction method for $P$ value, and the two-sided hypergeometric test was the enrichment/depletion..$^{15,16}$

\section{Results}

Two networks of interaction were constructed in this work, the first one without any additives and the second one with additional neighbors of 50 (Figures 1 and 2).

In the first network of $40 \mathrm{DEPs}$, there were 40 nodes with 108 interactions. Among these nodes, only 30 were connected. The other DEPs remained as individual nodes.

The second network consisted of the queried proteins plus the 50 neighbors, which was 90 nodes in total. This network had 1154 connections. Three of the queried proteins remained as individual nodes. These nodes were Clec3b, Nlrp10, and Phldb2 that did not come to the connection with other nodes. Further analysis based on network centrality showed that some of the proteins possessed valuable properties in the strength of the network. In Table 1, the list of the proteins with the highest centrality scores are gathered and ranked based on the degree value.

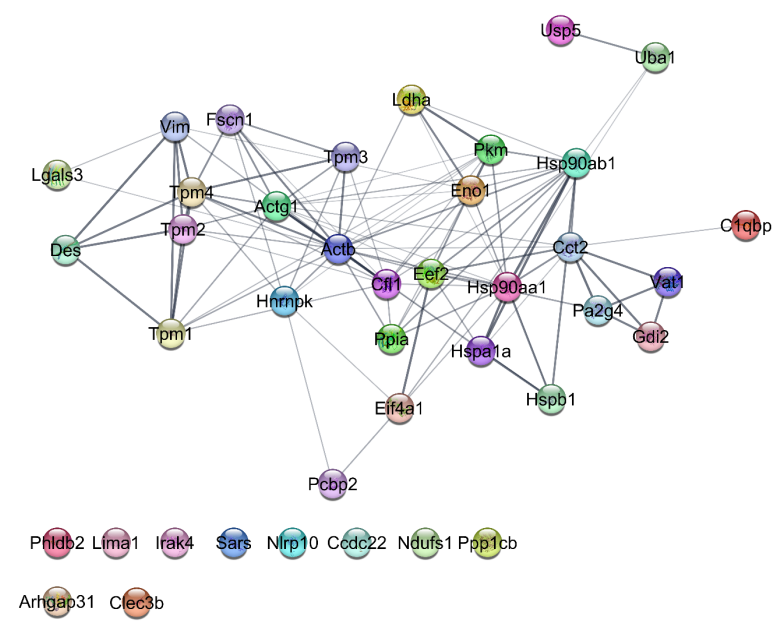

Figure 1. The Network of DEPs Query Without Any Additive Neighbors in a Cytoscape Platform. Numbers of 10 ones are isolated. The main connected component is formed via undirected connections.

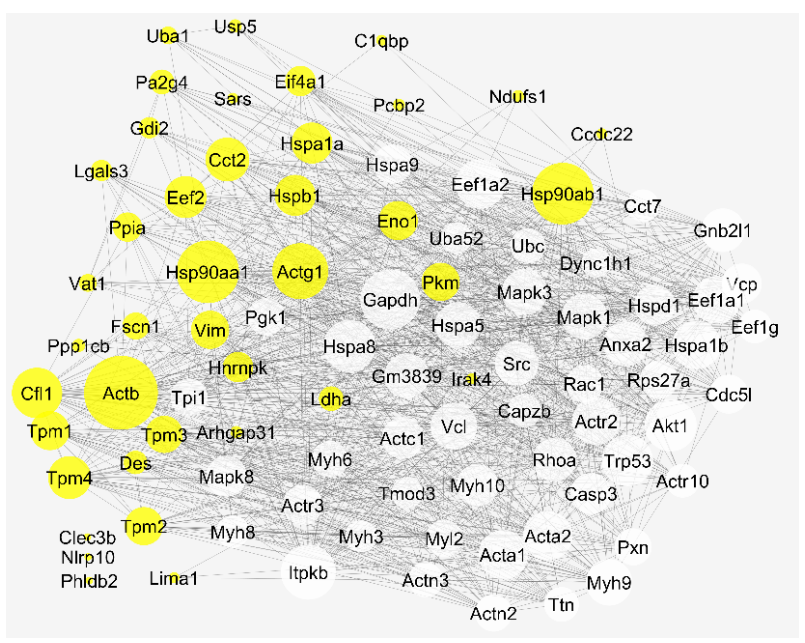

Figure 2. The Network of 40 Proteins With the 50 Added Neighbors. The query proteins are highlighted and the node size indicates the degree value analyzed by the Network Analyzer application of Cytoscape software. 
Table 1. The List of the Hub-Bottlenecks of the Network of IR Laser Treatment on the C2C12 Cell Line

\begin{tabular}{lcccc}
\hline Display Name & Accession ID & Expression Condition & K & BC \\
\hline Actb & Q6ZWM3 & Up-regulated & 65 & 0.06 \\
Hsp90aa1 & P07901 & Up-regulated & 53 & 0.03 \\
Hsp90ab1 & P11499 & Up-regulated & 52 & 0.03 \\
Gapdh* & P16858 & - & 50 & 0.02 \\
Actg1 & P63260 & Up-regulated & 46 & 0.02 \\
Itpkb* & Q9WV69 & - & 45 & 0.02 \\
\hline
\end{tabular}

aThe nodes with a star are from neighbor proteins in the network.

The centrality analysis of the second network showed that most of the hub-bottleneck proteins were from query DEPs. Only two were from neighbor proteins, which were Gapdh and Itpkb. Actb, the most highlighted hubbottleneck, was with a degree of 65 and betweenness centrality of 0.06 .

Biological processes related to the central nodes were provided by ClueGO. The terms are clustered and the identified groups are represented based on significant parameters in Figure 3.

Nitric oxide synthase (NOS) regulator activity was the leading biological process group with about $48 \%$ of the entire BPs in this query in ClueGO. The presence of 2 proteins per term was obtained for the hub-bottleneck DEPs. Likewise, HSP90aa1 and HSP90ab1 belonged to the NOS regulator activity; Actg1 and Actb were the related structural constituent of the postsynaptic actin cytoskeleton. The other 2 nodes which were not from differentially expressed proteins were not common in a term; however, Itpkb and Gapdh were linked to inositol1,4,5-trisphosphate 3-kinase activity and Peptidylcysteine 5-nintrosylase activity individually.

\section{Discussion}

Bioinformatics including protein-protein interaction network analysis could help in the light of understanding the mechanism in which the effect of applied treatment on living organisms is detected. Laser therapy, which has been used since the 1970s, is one of the common methods in muscle recovery treatments. ${ }^{4}$ Here, to better recognize the IR effectiveness in muscle cells, the DEPs from the original proteomics study of IR Laser treatment on myoblast cell were evaluated in silico. Two PPI networks were constructed from the merely queried proteins (DEPs) and the queried individuals plus 50 neighbor proteins. The condense interaction between these proteins and the other nodes indicated their important interaction values. Moreover, the first network query without any additives showed that the DEPs were in a cluster of interactions. The network centrality analysis implied the presence of essential nodes that were vital for network stability. These nodes were recognized as 6 central proteins in the network of IR laser treatment on the C2C12 cell line.

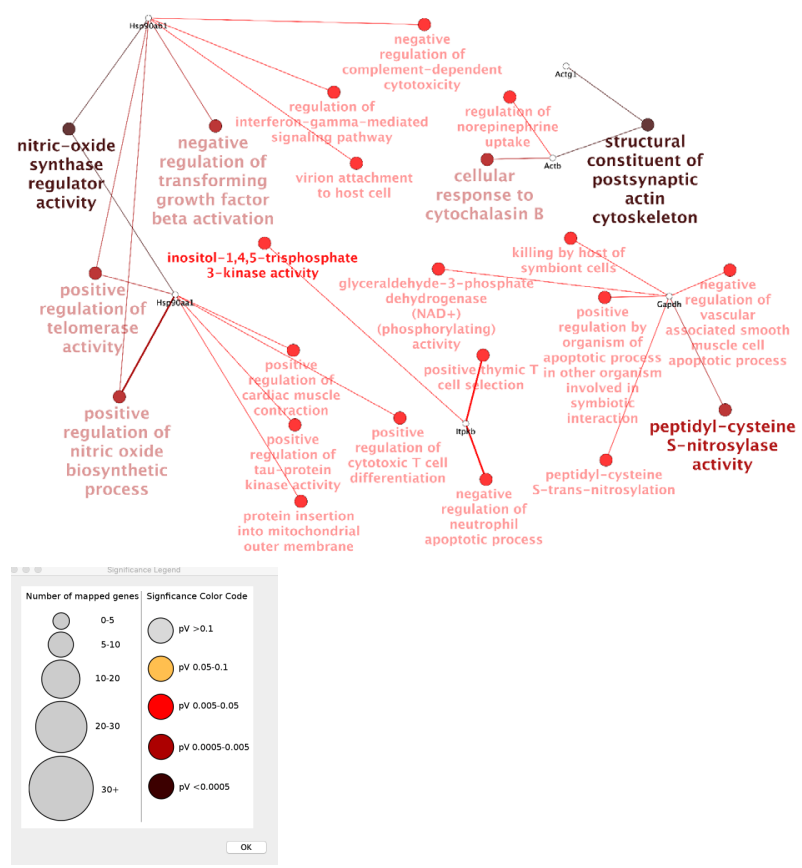

Figure 3. The Significance BP Map of Hub-Bottleneck Proteins, the Size, and the Color of Nodes Indicate Their Significance. The highlighted terms indicate their leadership in their corresponding group.

Among them, Actb, Hsp90aa1, Hsp90ab1, and Actg1 were the DE hub-bottlenecks. Gapdh and Itpkb were the other 2 hub-bottlenecks, but they were from the neighbor proteins. There were four biological processes linked to the DE central proteins and accordingly, NOS regulator activity and the structural constituent of the postsynaptic actin cytoskeleton could definitely get affected as a result of dysregulation of DE hub-bottlenecks.

Chaperons are protein molecules that bind to the non-native proteins to obtain biological function. These molecular machines are overexpressed in the stress condition and protect the body. ${ }^{17,18}$ Two kinds of Hsp90a (50\% of queried central DEPs), namely Hsp90aal and Hsp90ab1, are highlighted as hub-bottlenecks with similar centrality parameters (see Table 1). The up-regulation of HSPs was also referred to by the original study, which has to do with promoting cell differentiation. This process was reported as being accelerated by the application of IR laser in muscle recovery. ${ }^{4}$ The HSP90s as the central nodes could have great participation in cell differentiation after laser application. The regulatory linkage of HSP90s and NOS was stated in this study and the up-regulation of HSP90s could contribute to the NOS activation which was also previously reported. ${ }^{19}$ The NOS activity is important for muscle healing ${ }^{20}$ and the findings show that it could be promoted by laser application since the HSP90s expression increases.

The well-known molecular mechanism of muscle contraction is tied to the interaction between actin and myosin filaments. ${ }^{21}$ Different variants of actin molecules, including alpha-actin, beta- actin, and gamma actin, are 
identified and involved in muscle contraction and also other vital cellular functions such as proliferation and synthetic or secretory activities. ${ }^{22}$ Since the overexpression of Actb and actg1 (the other $50 \%$ of queried DEPs) is associated with the repair procedure in muscle recovery, ${ }^{23}$ highlighting these two variants of actin via network analysis reveals their important role by laser radiation. It can be concluded that the IR laser can be considered as repair promotion and cell protection in different muscle stress conditions. The expression change of actin after low-intensity IRs radiation in skin and muscle is reported by Fonseca et al. ${ }^{24}$ In this document, it is reported that actin mRNA expression changes, depending on fluency, frequency and time of its exposure to radiation in the skin and muscle of Wistar rats.

Although the expression changes of the other 2 hubbottlenecks are not reported in the original study, they might have some roles in the underlying laser molecular mechanism. Glyceraldehyde-3-phosphate dehydrogenase, Gapdh, is known as a metabolic enzyme that is involved in sugar metabolism. The role of Gapdh in maintaining adequate glycolytic flux and related pathways are discussed by Seidler. ${ }^{25}$ Its character in muscle recovery and its function in stress condition and also after laser therapy have been reported by many studies. ${ }^{26,27}$ Its participation as a central protein in this study refers to a possible critical role of the IR laser in the evocation of cell metabolism. Itpkb, inositol trisphosphate 3-kinase B, as another neighbor hub-bottleneck in the PPI network, has been shown as a pathogenic factor for Alzheimer disease by increasing the level of amyloid beta in patients. ${ }^{28}$ Therefore, as a novel central protein in our network, it is vital to assess its feasible alterations as a possible side effect of laser treatments. Altogether, the central nodes and their corresponding biological processes could pinpoint the molecular mechanism of IR laser effectiveness.

\section{Conclusion}

IR Laser therapy is an effective method to promote muscle repairing by influencing central proteins of the PPI network of the C2C12 Cell Line. One of the crucial mechanisms could be through the activation of NOS. The Gapdh and Itpkb, regardless of not being DEPS, could also show potential in laser treatments; nevertheless, an intensive investigation is required for this claim.

\section{Ethical Considerations}

Not applicable.

\section{Conflict of Interests}

The authors declare no conflict of interest.

\section{Acknowledgment}

The Proteomics Research Center of Shahid Beheshti University of Medical Sciences supported this research.

\section{References}

1. Depfenhart M, Müller J, inventors; Telesto GmbH, assignee. Laser therapy system with UVA and IR laser light for directional generation of a dermal collagen matrix. United States patent US 9,486,284; November 8, 2016.

2. Shazly TA, Latina MA, inventors; VISUMEDICS Inc, assignee. Diagnostic and surgical laser device utilizing a visible laser diode. United States patent application US 10/016,302; July 10, 2018.

3. Canis M, Ihler F, Martin A, Matthias C, Steiner W. Transoral laser microsurgery for T1a glottic cancer: review of 404 cases. Head Neck. 2015;37(6):889-95. doi: 10.1002/ hed.23688.

4. Monici M, Cialdai F, Ranaldi F, Paoli P, Boscaro F, Moneti G, et al. Effect of IR laser on myoblasts: a proteomic study. Mol Biosyst. 2013;9(6):1147-61. doi: 10.1039/c2mb25398d.

5. Ceylan Y, Hizmetli S, Siliğ Y. The effects of infrared laser and medical treatments on pain and serotonin degradation products in patients with myofascial pain syndrome. A controlled trial. Rheumatol Int. 2004;24(5):260-3. doi: 10.1007/s00296-003-0348-6.

6. Fried D, Zuerlein M, Featherstone JDB, Seka W, Duhn C, McCormack SM. IR laser ablation of dental enamel: mechanistic dependence on the primary absorber. Appl Surf Sci. 1998;127-129:852-6. doi: 10.1016/S01694332(97)00755-1.

7. Silveira PC, Scheffer Dda L, Glaser V, Remor AP, Pinho RA, Aguiar Junior AS, et al. Low-level laser therapy attenuates the acute inflammatory response induced by muscle traumatic injury. Free Radic Res. 2016;50(5):50313. doi: 10.3109/10715762.2016.1147649.

8. Rezaei-Tavirani M, Tavirani MR, Azodi MZ, Farshi HM, Razzaghi MR. Evaluation of skin response after erbium: yttrium-aluminum-garnet laser irradiation: a network analysis approach. J Lasers Med Sci. 2019;10(3):194-99. doi:10.22037/jlms.v10i3.24698.

9. Safaei A, Rezaei Tavirani M, Zamanian Azodi M, Lashay A, Mohammadi SF, Ghasemi Broumand M, et al. Diabetic retinopathy and laser therapy in rats: A proteinprotein interaction network analysis. J Lasers Med Sci. 2017;8(1):S20-21. doi: 10.15171/jlms.2017.s4.

10. Rostami A, Shahani M, Zarrindast MR, Semnanian S, Rahmati Roudsari M, Rezaei Tavirani M, et al. Effects of $3 \mathrm{~Hz}$ and $60 \mathrm{~Hz}$ extremely low frequency electromagnetic fields on anxiety-like behaviors, memory retention of passive avoidance and electrophysiological properties of male rats. J Lasers Med Sci. 2016;7(2):120-5. doi: 10.15171/ jlms.2016.20.

11. Szezerbaty SKF, de Oliveira RF, Pires-Oliveira DAA, Soares CP, Sartori D, Poli-Frederico RC. The effect of low-level laser therapy $(660 \mathrm{~nm})$ on the gene expression involved in tissue repair. Lasers Med Sci. 2018;33(2):315-21. doi: 10.1007/s10103-017-2375-7.

12. Rezaie-Tavirani M, Hasanzadeh H, Seyyedi S, Zali H. Proteomic analysis of the effect of extremely low-frequency electromagnetic fields (ELF-EMF) with different intensities in SH-SY5Y neuroblastoma cell line. J Lasers Med Sci. 2017;8(2):79-83. doi: 10.15171/jlms.2017.14.

13. Shannon P, Markiel A, Ozier O, Baliga NS, Wang JT, Ramage $\mathrm{D}$, et al. Cytoscape: a software environment for integrated models of biomolecular interaction networks. Genome Res. 
2003;13(11):2498-504. doi: 10.1101/gr.1239303.

14. Szklarczyk D, Franceschini A, Kuhn M, Simonovic M, Roth A, MinguezP, etal. The STRING database in 2011: functional interaction networks of proteins, globally integrated and scored. Nucleic Acids Res. 2011;39(1):D561-D568. doi: 10.1093/nar/gkq973.

15. Bindea G, Galon J, Mlecnik B. CluePedia Cytoscape plugin: pathway insights using integrated experimental and in silico data. Bioinformatics. 2013;29(5):661-3. doi: 10.1093/ bioinformatics/btt019.

16. Bindea G, Mlecnik B, Hackl H, Charoentong P, Tosolini M, Kirilovsky A, et al. ClueGO: a Cytoscape plug-in to decipher functionally grouped gene ontology and pathway annotation networks. Bioinformatics. 2009;25(8):1091-3. doi: 10.1093/bioinformatics/btp101.

17. Bukau B, Horwich AL. The Hsp70 and Hsp60 chaperone machines. Cell. 1998;92(3):351-66. doi: 10.1016/s00928674(00)80928-9.

18. Mosser DD, Caron AW, Bourget L, Meriin AB, Sherman MY, Morimoto RI, et al. The chaperone function of hsp70 is required for protection against stress-induced apoptosis. Mol Cell Biol. 2000;20(19):7146-59. doi: 10.1128/ mcb.20.19.7146-7159.2000.

19. Harris MB, Mitchell BM, Sood SG, Webb RC, Venema RC. Increased nitric oxide synthase activity and Hsp90 association in skeletal muscle following chronic exercise. Eur J Appl Physiol. 2008;104(5):795-802. doi: 10.1007/ s00421-008-0833-4.

20. Anderson JE. A role for nitric oxide in muscle repair: nitric oxide-mediated activation of muscle satellite cells. Mol Biol Cell. 2000;11(5):1859-74. doi: 10.1091/mbc.11.5.1859.

21. Rayment I, Holden HM, Whittaker M, Yohn CB, Lorenz M, Holmes KC, et al. Structure of the actin-myosin complex and its implications for muscle contraction. Science.
1993;261(5117):58-65. doi: 10.1126/science.8316858.

22. Fatigati V, Murphy RA. Actin and tropomyosin variants in smooth muscles. Dependence on tissue type. J Biol Chem. 1984;259(23):14383-8

23. Pilegaard H, Ordway GA, Saltin B, Neufer PD. Transcriptional regulation of gene expression in human skeletal muscle during recovery from exercise. Am J Physiol Endocrinol Metab. 2000;279(4):E806-14. doi: 10.1152/ ajpendo.2000.279.4.E806.

24. Fonseca AS, Mencalha AL, Campos VMA, FerreiraMachado SC, Peregrino AAF, Magalhães LAG, et al. Lowintensity infrared lasers alter actin gene expression in skin and muscle tissue. Laser Phys. 2012;23(2):025602. doi: 10.1088/1054-660X/23/2/025602.

25. Seidler NW. GAPDH: Biological Properties and Diversity. The Netherlands: Springer; 2013. p. 37-59.

26. Mesquita-Ferrari RA, Martins MD, Silva JA Jr, da Silva TD, Piovesan RF, Pavesi VCS, et al. Effects of low-level laser therapy on expression of TNF- $\alpha$ and TGF- $\beta$ in skeletal muscle during the repair process. Lasers Med Sci. 2011;26(3):335-40. doi: 10.1007/s10103-010-0850-5.

27. Corona BT, Machingal MA, Criswell T, Vadhavkar M, Dannahower AC, Bergman C, et al. Further development of a tissue engineered muscle repair construct in vitro for enhanced functional recovery following implantation in vivo in a murine model of volumetric muscle loss injury. Tissue Eng Part A. 2012;18(11-12):1213-28. doi: 10.1089/ ten.TEA.2011.0614.

28. Stygelbout V, Leroy K, Pouillon V, D’Amico E, Erneux C, Schurmans $\mathrm{S}$, et al. Overexpression of inositol 1, 4, 5-trisphosphate 3-kinase B (Itpkb) in neurons of APP mice leads to an increase of Amyloid Beta production. Alzheimers Dement 2011;7(4):S528-S9. doi: 10.1016/j. jalz.2011.05.1483. 